Article

\title{
Statistical Technology Analysis for Competitive Sustainability of Three Dimensional Printing
}

\author{
Sangsung Park ${ }^{1}$ and Sunghae Jun ${ }^{2, *}$ \\ 1 Graduate School of Management of Technology, Korea University, Seoul 136-701, Korea; hanyul@korea.ac.kr \\ 2 Department of Statistics, Cheongju University, Chungbuk 360-764, Korea \\ * Correspondence: shjun@cju.ac.kr; Tel.: +82-10-7745-5677; Fax: +82-43-229-8432
}

Academic Editor: Marc A. Rosen

Received: 21 April 2017; Accepted: 21 June 2017; Published: 29 June 2017

\begin{abstract}
The technology of three-dimensional (3D) printing was commercialized in the late 1980s. Since then, the development of this technology has been dramatically increasing. Moreover, 3D printing technology has been used in many different fields, such as electronics and medical appliances, because 3D printing is a technological convergence based on precision instruments, chemical materials, and electrical equipment. The technological impact of 3D printing is so powerful that we need to analyze 3D printing technology to understand the 3D printing industry. In addition, we want more analytical results for understanding the sustainability of 3D printing technology. Thus, we compare the technologies between 3D printing competitors to find their technological innovations and evolution from a technological sustainability. To analyze the 3D printing technology, we propose a new methodology of statistical technology analysis combing social network analysis with time series clustering. In our case study, we make a comparison between "3D Systems" and "Stratasys", two major 3D printing companies, because they have been leading the sustainable technologies of $3 \mathrm{D}$ printing in the market. We illustrate how the proposed methodology can be applied to practical problems from the case study. This paper contributes to the sustainable technology management, and our research can expand to other competitors with diverse technological fields as well as 3D printing.
\end{abstract}

Keywords: sustainable technology; statistical technology analysis; three-dimensional printing; social network analysis; time series clustering

\section{Introduction}

Research and development of sustainable technologies is a very important issue in the present situation. Development of sustainable technologies in various fields such as climate change, food shortage, and renewable energy has become necessary. This is because we have to solve these human problems through sustainable technology development. Sustainable technology means technology that solves many problems that arise in order to sustain human life. That is, sustainable development of society depends on sustainable technology. However, it is difficult to assess the sustainability of a technology, and there are still no general measures to evaluate sustainability for technology [1]. In addition, sustainability is very important to the management of technology (MOT), and a company with sustainable technology is competitive in the markets [2]. Thus, most companies have tried to develop and keep the sustainable technologies in their areas. In addition, the studies on the sustainable technology were performed in diverse domains [3-7]. A number of studies have been conducted to determine the sustainability of technology. Kim et al. (2015) proposed a methodology for sustainable management of defense technology [3]. They studied on growth curves based on expert survey and patent data to assess the sustainability of defense technology. A research on the selection method of sustainable technology was introduced by Choi et al. (2015) [4]. The authors used a network model based on social network analysis (SNA) for sustainable technology selection, and chose 
sustainability as the technology with the largest centrality in SNA results. In addition, Choi et al. (2016) constructed a hierarchical diagram of technology for understanding the causal relations between representative technologies in telematics technology, and they extracted the sustainable technologies from the technological relationships for telematics [6]. Lastly, Kim et al. (2016) proposed a hybrid patent analysis method for managing sustainable technology [7]. They also selected the field of humanoid robot technology as their target technology, and found a sustainable technology for this technology using the proposed method. In the three-dimensional (3D) printing, some enterprises are in competition with each other to get the sustainable technology of 3D printing. We consider the 3D printing technology as a domain of sustainable technology analysis in this paper. The 3D printing technology is technology to make a target product by piling up various materials [8]. This technology was commercialized in the late 1980s, and since then, has been developing dramatically in diverse fields such as electronics, vehicles, medical appliances, office products, art, architecture, and entertainment. Most governments and companies have mentioned that $3 \mathrm{D}$ printing is an emerging technology that could change the world. 3D printing technology can make any product with any shape at low cost. This is creating huge revolution in various areas such as manufacturing and medicine. In addition, the technological impact of 3D printing is very powerful in our everyday lives. However, the commercial marketplace for 3D printing is still in its early stages. Accordingly, the 3D printing technology shows great potential to technological sustainability. The market of 3D printing has been dominated by two leading companies. As of 2012, the market share for industrial 3D printers was as follows: Stratasys was in first place, and 3D Systems was in second [9]. The two companies together had a majority of the market share. Further, until August 2013, 3D Systems and Stratasys were the leading companies with the most published and issued patents related to 3D printing [8]. To understand and prepare for the era of the 3D printing from the perspective of sustainability, we need to analyze the technologies held by Stratasys and 3D Systems. In this paper, we compare the technologies of these two competitors to determine the technological innovation and evolution of 3D printing technology. We analyze the patent documents related to 3D printing technology. Many researches for technology analysis have used the patent data because a patent contains meaningful and rich information about developed technology [10-13]. To analyze 3D printing technology, we propose a methodology of patent analysis combined with social network analysis (SNA) and time series clustering (TSC). Many researches related to patent analysis by SNA have been published in the MOT fields [14-17]. These studies used forecasting future technology, extracting core technology, or building a technological relationship using SNA graphs and its centrality measures $[17,18]$. Recently, TSC was used as a useful method for patent technology analysis [19]. Many studies on text and document analyses as well as patent document have been performed in diverse fields [20-22]. They are also useful for patent analysis. It is important to analyze patent documents for technology analysis because a patent contains most of the results of developed technology. In this paper, we combine SNA and TSC to construct an analytical method for technological comparison between competitors. Further, in our case study, we compare Stratasys and 3D Systems using the proposed methodology. Using the results of case study, we will provide the technological levels of two companies for their sustainability. Thus, we show how the proposed methodology can be applied to the real 3D printing domain. Moreover, most studies of technology analysis of competitors were depended on the knowledge and experience from the domain experts and descriptive statistics. However, we need additional information to improve the performance of technology analysis of competitors. To solve this problem, we propose a methodology of patent analysis based on advanced statistical methods. The originality of this paper is to show novel approach to compare the technological sustainability between competitors. The remainder of this paper is organized as follows. Section 2 introduces text mining and SNA for patent analysis. In our research, we apply SNA and TSC to a combined model for technology analysis of two leading companies related to 3D printing, and explain more detailed technology analysis of 3D printing technology between competitors in Section 3. In next section, we perform a case study to illustrate how the proposed 
could be used in real problems. We discuss the research results and present our conclusions in the last section.

\section{Statistical Approach to Patent Analysis}

In MOT fields, it is important to analyze technology for sustainable technology, R\&D planning, competitors' analysis, etc. [2,6,23-26]. In this paper, we apply statistical modeling to technology analysis between the competitive companies of Stratasys and 3D Systems in 3D printing domain. In particular, we compare them in terms of sustainable technology. Many researches for technology analysis were based on patent analysis [11-13,15,27-30] because the patent contains complete results of developed technologies [2,31]. However, we cannot analyze the patent documents directly using statistical modeling. In general, patent data are not suitable for statistical analysis [24]. Thus, we have to preprocess the retrieved patent documents for statistical modeling. We also use patent document data for technology analysis in this paper. The first point to be considered in patent document analysis is text mining for preprocessing patent data because patent documents are not suitable for quantitative analyses based on statistics [17,32-35]. Thus, we transform the patent documents into structured data for statistical modeling. Text mining is to discover hidden and novel patterns from text document data [36]. In addition, text mining is interdisciplinary of statistics, computer science, and linguistics, and performs text data analysis using the theoretical and practical methods from the interdisciplinary fields. In patent analysis, a major role of text mining is to transform the patent documents into structured data. Figure 1 shows this process.

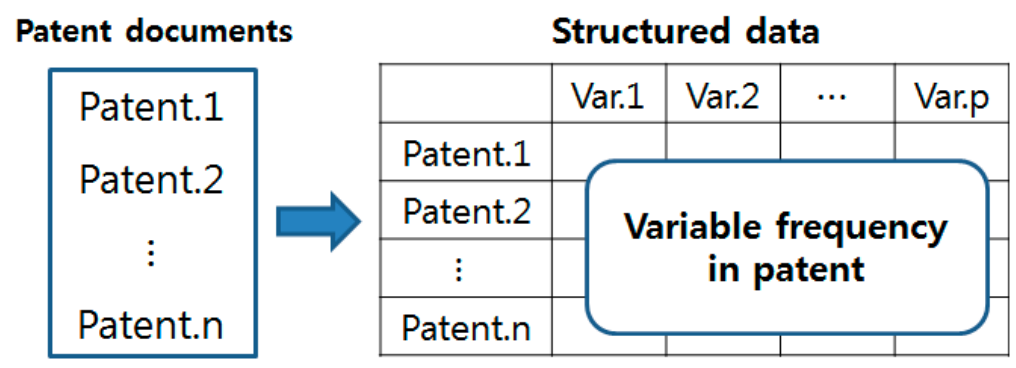

Figure 1. Transforming patent documents into structured data using text mining.

The structured data consist of row and column in a table. The row and column represent patent and variable respectively, and each cell of the table is variable frequency in each patent. This table (structured data) is suitable for statistical analysis. In addition, in patent analysis, the variable can become keyword or IPC (international patent classification) code. In our research, we use this structured data to carry out SNA and TSC methods for technology analysis. SNA is based on a graph data structure as follows [16,18,37].

$$
\begin{gathered}
\text { Nodes }=\left\{\mathrm{N}_{1}, \mathrm{~N}_{2}, \ldots, \mathrm{N}_{\mathrm{p}}\right\} \\
\text { Connections }=\{\mathrm{C}(\mathrm{N} 1, \mathrm{~N} 2), \mathrm{C}(\mathrm{N} 1, \mathrm{~N} 3), \ldots, \mathrm{C}(\mathrm{Np}-1, \mathrm{~Np})\}
\end{gathered}
$$

Nde $N_{i}$ is a social object in a social system, and the connection $C\left(N_{i}, N_{j}\right)$ represents a binary relation between $\mathrm{N}_{\mathrm{i}}$ and $\mathrm{N}_{\mathrm{j}}$. If a meaningful connection exists, the value of $C\left(\mathrm{~N}_{\mathrm{i}}, \mathrm{N}_{\mathrm{j}}\right)$ is one; otherwise, it is zero. Thus, the SNA is used to analyze the relationship between nodes arising from social systems. In this paper, we use technological keywords as the node of SNA below.

$$
\text { Technology nodes }=\left\{\operatorname{Keyword}_{1}, \operatorname{Keyword}_{2}, \ldots, \text { Keyword }_{\mathrm{p}}\right\}
$$

Technological connections $=\{C($ Keyword1, Keyword2 $), \ldots, C($ Keywordp-1, Keywordp $)\}$ 
We extract the keywords from patent documents, and they contain meaningful information for developed technologies. We can find the technological relations from the SNA graph based on the technology keywords. In our research, we consider both connections to determine the technological relations among all technologies. The results of SNA are combined with TSC results to analyze the sustainable technology in this research.

\section{Statistical Technology Analysis for Competitive Sustainability}

However, the previous works related to sustainable technology did not deal with the time periods of technology developments. Consideration of time in sustainable technology is an important factor, because sustainability involves the concept of time. In order to solve this problem, we have incorporated the results of the changes in technology over time into the analysis model to find sustainable technology. We attempted to grasp trends of technology keywords according to time and to classify similar technology keywords into the clusters. In our research, we carry out time series clustering (TSC) based on the measure of dynamic time warping (DTW) and social network analysis (SNA) graph to find the sustainability in 3D printing technology. 3D printing technology is revolutionizing the manufacturing industry due to breakthrough technology development such as development of information and communication technology and software, open source community culture, material engineering. Since the late 2000s, this technology has attracted worldwide attention as a promising and emerging technology. D'Aveni (2015) said that 3D printing technology is at the beginning of a revolution in the manufacturing industry around the world [38].

To construct a methodology for sustainable technology analysis, we combine the TSC with text mining and SNA described in the previous section. We compare two competitors based on their technological evolution using the proposed methodology and find meaningful relations for their sustainable technologies. First, we consider the frequency trend of the technological keywords over a year as the time series (TS) data. Time series analysis is to analyze time series data for decomposition, classification, and clustering [39]. The main goal of this analysis is to forecast future trend of time series. In this paper, the time series data are the frequency of patent keywords over year. We construct a TSC model of patent keywords for comparing the technological competition between 3D Systems and Stratasys. In addition, we apply the dynamic time warping (DTW) to make our TSC model. Among the diverse methods of TSC, we use the DTW. This is used to cluster TS data into similar groups using distance measures [40-42]. In this paper, we assign the keywords to similar clusters using DTW. Figure 2 shows the concept of DTW clustering.

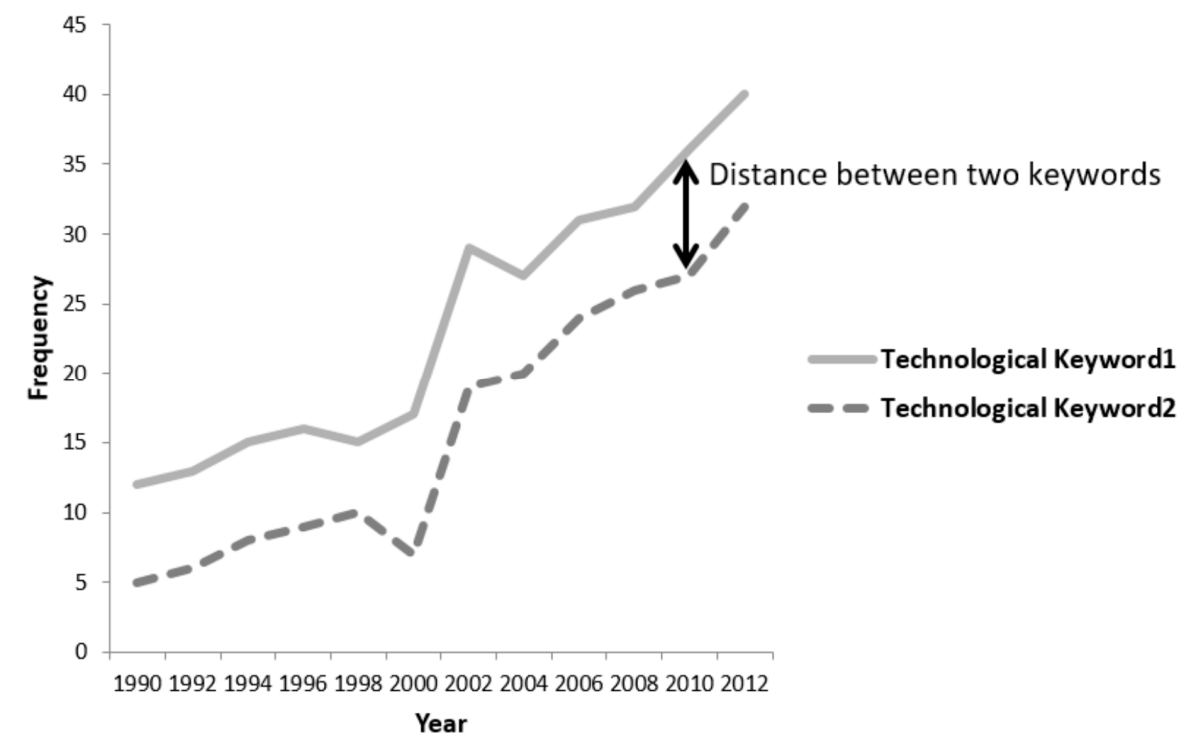

Figure 2. Distance between time series trends. 
The DTW computes the distance values among two technological keywords based on their TS trends, and finds the optimal alignment between the TS trends [43,44]. Figure 2 shows two TS trends: technological keyword 1 (solid line) and technological keyword 2 (dotted line). The DTW compares paired peak and valley points one after another $[43,44]$. Based on this process of DTW, we cluster all keywords into similar clusters using a hierarchical clustering approach. Given technological keywords $i$ and $j(i, j=1,2, \ldots, p$ and $i \neq j)$, we get the optimal DTW value by minimizing distance between two keywords as follow.

$$
\text { Distance }_{\text {optimal }}=\text { arg.min.dist }_{\left(\text {keyword }_{i}, \text { keyword }_{j}\right)}
$$

In the DTW, the distance means total distance between aligned vectors of technology keyword $i$ and $j$. TSC is to separate the patent keywords into similar groups based on distance measure. Among various measures such as Euclidean distance and inner product, the DTW showed good performance for TSC in technology analysis [19]. The DTW proposed to overcome the limitation of Euclidean distance by providing an intuitive distance between time series data [45]. Given time series $X$ and $Y$, where $X$ and $Y$ represent two patent keywords, are shown as follow [42]; $X=\left(x_{1}, x_{2}, \ldots, x_{n}\right)$ and $Y=\left(y_{1}, y_{2}, \ldots, y_{m}\right)$. We build a warp path $W$ below.

$$
W=\left(w_{1}, w_{2}, \ldots, w_{k}\right) \text {, where, } \max (n, m) \leq k \leq n+m
$$

The $k$ shows the length of warp path. We can the similarity between $X$ and $Y$ by $W$. In this paper, we use the data matrix for the TSC based on DTW in Figure 3.

\begin{tabular}{|c|c|c|c|c|}
\hline Year & Keyword.1 & Keyword.2 & $\cdots$ & Keyword.p \\
\hline $\mathbf{1}$ & \multirow{3}{*}{ Time series data } \\
\hline $\mathbf{2}$ & \multirow{3}{*}{ Time series } \\
\hline$\vdots$ & & \\
\hline $\mathbf{T}$ & &
\end{tabular}

Figure 3. Time series data matrix for TSC based on DTW.

The elements of this matrix are occurred frequency of the keywords by year. Using the time series data matrix, we compute DTW distances between two keywords, and get the DTW distance matrix in Figure 4.

\begin{tabular}{|c|c|c|c|c|}
\hline & Keyword.1 & Keyword.2 & $\cdots$ & Keyword.p \\
\hline Keyword.1 & Dist $\left(\mathrm{W}_{11}\right)$ & Dist $\left(\mathrm{W}_{12}\right)$ & & Dist $\left(\mathrm{W}_{1 \mathrm{p}}\right)$ \\
\hline Keyword.2 & Dist $\left(\mathrm{W}_{21}\right)$ & Dist $\left(\mathrm{W}_{22}\right)$ & & Dist $\left(\mathrm{W}_{2 \mathrm{p}}\right)$ \\
\hline$\vdots$ & & & & \\
\hline Keyword.p & Dist $\left(\mathrm{W}_{\mathrm{p} 1}\right)$ & $\operatorname{Dist}\left(\mathrm{W}_{\mathrm{p} 2}\right)$ & & Dist $\left(\mathrm{W}_{\mathrm{pp}}\right)$ \\
\hline
\end{tabular}

Figure 4. DTW distance matrix of patent keywords.

In Figure 4, $\operatorname{Dist}\left(W_{i j}\right)$ is the computation result of DTW distance between keywords $i$ and $j$. In Figure 5, we construct a hierarchical TSC diagram of patent keywords.

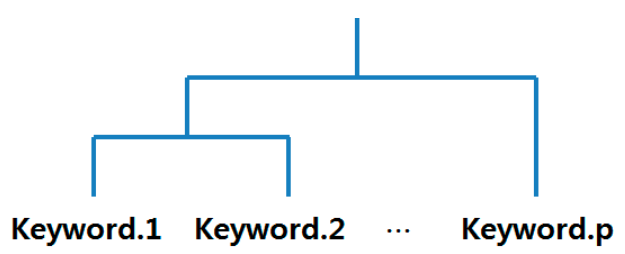

Figure 5. Hierarchical TSC diagram by DTW distance matrix. 
We can find the technological association of a company using this hierarchical diagram. Using this diagram, we can assign each keyword into one of clusters being considered time. We use another approach to analysis of competitor technology using the SNA. By the text mining and keyword extraction, we construct the patent-keyword matrix in Figure 6 for performing SNA.

\begin{tabular}{|c|c|c|c|c|}
\hline & Keyword.1 & Keyword.2 & $\cdots$ & Keyword.p \\
\hline Patent.1 & & & & \\
\hline Patent.2 & \multirow{3}{|}{ Keyword frequency in patent } \\
\hline$\vdots$ & & \\
\hline Patent.n & & \\
\hline
\end{tabular}

Figure 6. Patent-keyword matrix.

The elements of this matrix consist of the occurred frequencies of keywords in patents. Next, in Figure 7, we compute correlation coefficient values of the keywords, and construct keyword correlation matrix.

\begin{tabular}{|c|c|c|l|c|}
\hline & Keyword.1 & Keyword.2 & $\cdots$ & Keyword.p \\
\hline Keyword.1 & $\operatorname{Corr}(1,1)$ & $\operatorname{Corr}(1,2)$ & & $\operatorname{Corr}(1, p)$ \\
\hline Keyword.2 & $\operatorname{Corr}(2,1)$ & $\operatorname{Corr}(2,2)$ & & $\operatorname{Corr}(2, p)$ \\
\hline$\vdots$ & & & & \\
\hline Keyword.p & $\operatorname{Corr}(p, 1)$ & $\operatorname{Corr}(p, 2)$ & & $\operatorname{Corr}(p, p)$ \\
\hline
\end{tabular}

Figure 7. Keyword correlation matrix.

Using this matrix, we get adjacent matrix for building SNA graph. In this process, we should determine the cut-off value, and apply it to keyword correlation matrix. If the correlation value is larger than the cut-off value, the binary value of adjacent matrix is one. Otherwise, the binary value is zero in Figure 8.

\begin{tabular}{|c|c|c|c|c|}
\hline & Keyword.1 & Keyword.2 & $\cdots$ & Keyword.p \\
\hline Keyword.1 & binary $(1,1)$ & $\operatorname{binary}(1,2)$ & & binary $(1, p)$ \\
\hline Keyword.2 & $\operatorname{binary}(2,1)$ & $\operatorname{binary}(2,2)$ & & binary $(2, p)$ \\
\hline$\vdots$ & & & & \\
\hline Keyword.p & binary $(p, 1)$ & binary $(p, 2)$ & & binary $(p, p)$ \\
\hline
\end{tabular}

Figure 8. Adjacent matrix for SNA.

In this paper, we make SNA graph using the adjacent matrix and compare the technology of two competitive companies by SNA graph. Therefore, we study on a methodology based on text mining, SNA and DTW for technology analysis of the 3D printing competitors. From the results of technology analysis between the competitors, we can manage technology efficiently for technological sustainability between competitors, because sustainable technology is a key factor in MOT within a company [27,35,45-47]. Sustainability is a very important issue in technology management area [2]. A company finds and understands its sustainable technology to improve its technological competition. In addition, a company should adopt its sustainable technology to develop new technologies and to launch innovative products. In our research, we use R data language and its packages "tm", "sna", 
and "dtw" for patent analysis based on the proposed methodology [33,36,43,48-50]. We also collect the patent document data by searching from world patent databases $[2,31,51,52]$. The step-by-step procedures of proposed methodology are shown below.

Step 1. Collect patent documents applied by Stratasys and 3D Systems from patent databases.

Step 2. Make structured patent data using text mining techniques.

Step 3. Extract keywords from the structured patent data.

Step 4. Analyze patent data to understand sustainable technology.

4-1. Perform patent keyword clustering using TSC based on DTW.

4-2. Construct SNA graphs of patent keywords.

Step 5. Test the result of the patent analysis.

Step 6. Combine the results of TSC and SNA of technological keywords.

Step 7. Compare sustainable technologies between competitors.

Lastly, we inspect the statistical validity of SNA graph statistics using the conditional uniform graph tests $[37,49]$. We use this test as a method to assess the sustainability of the patent keyword. We compare two SNA graphs of Stratasys and 3D Systems by their test statistics (test values). The sustainable technology holds enormous implication for technological development and management. Thus, we extract the sustainable technologies from two companies (Stratasys and 3D Systems), compare their sustainable technologies being depended on the patent keywords, and conclude the technological strategies of two companies. In the next section, we carry out a case study of two competitors related to 3D printing technology using the proposed methodology. This shows how our work could be applied to practical areas.

\section{Case Study of Two Competitors}

To show how the proposed methodology could be applied to real problem, we compared two competitors of 3D printing technology. "Stratasys" and "3D Systems" are the top two companies in 3D printing industry. They have competed for the lead in market share and patent applications of developed technologies, and developed and maintained the competitive sustainability of 3D printing technology. Many analytical reports have dealt with the two competitors $[8,53,54]$. For our case study, we collected all patents applied for by Stratasys and 3D Systems to 2012. 3D Systems applied for 538 patents and registered 314 patents, while Stratasys applied for 270 patents and registered 138. Figure 9 shows the numbers of patents applied by two companies by year.

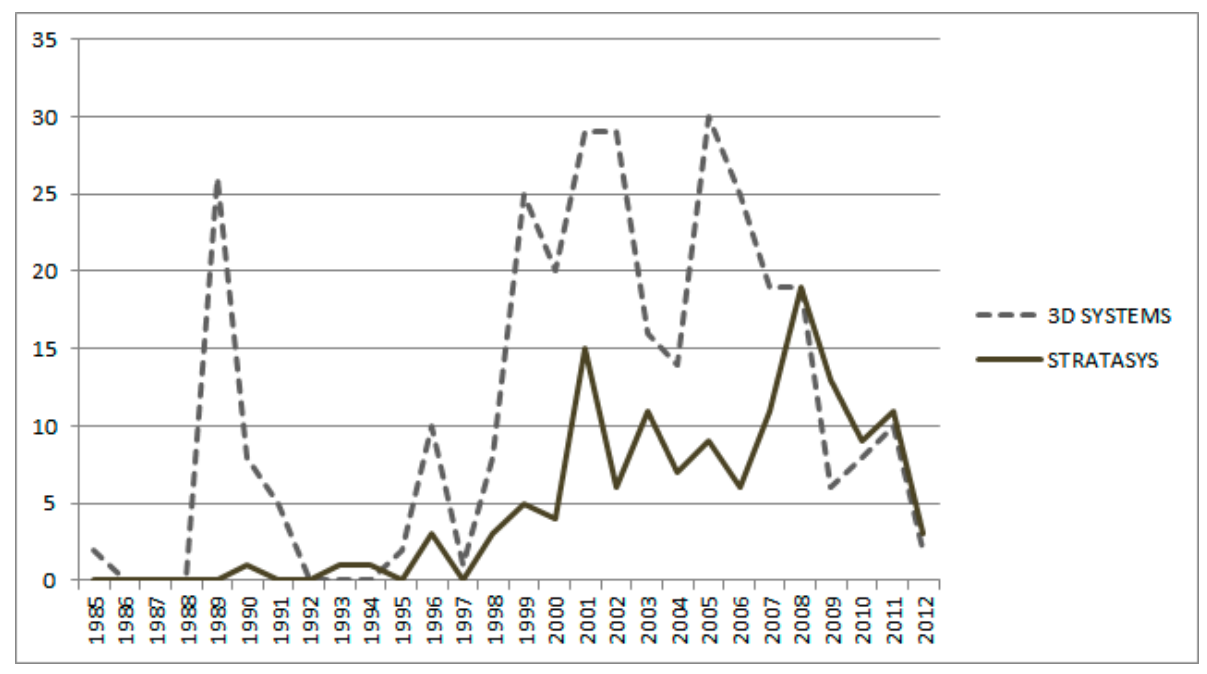

Figure 9. Annual number of patents applied by two competitors. 
3D Systems registered two patents for the first time in 1985, while Stratasys registered its first patent in 1990. At the beginning of the 2000s, 3D Systems was superior to Stratasys in terms of registered patents relating to 3D printing technology, but Stratasys got the jump on 3D Systems in the late 2000s. Overall, although 3D Systems holds more than double the number of patents as Stratasys, Stratasys is displaying a tendency to give chase to 3D Systems. In this paper, we analyzed the patent data from the two companies using SNA and TSC. First, we extract the keywords from the patents of the two companies. In this case study, we removed common and meaningless words such as "a", "is", and "and", and we selected keywords with high frequency and relevance to 3D printing technology with the help of the 3D printing expert at the Korea Intellectual Property Strategy Agency (KISTA) [55]. Finally, we determined 32 and 20 keywords to represent 3D Systems and Stratasys technologies respectively. We divided the keywords into common and representative keywords for the two companies in Table 1.

Table 1. Extracted keywords of two competitors.

\begin{tabular}{|c|c|c|}
\hline Company & Common Keywords & Representative Keywords \\
\hline 3D Systems & \multirow{2}{*}{$\begin{array}{l}\text { Method, Modeling, System, } \\
\text { Three_Dim, Manufacturing, Rapid, } \\
\text { Building, Deposition, Material, } \\
\text { Object, Support, Printing }\end{array}$} & $\begin{array}{l}\text { Laser, Selective, Solid, Pour, Composition, Forming, } \\
\text { Production, Control, Imaging, Sintering, Phase, } \\
\text { Technique, Chamber, Stereolithography, Appareil, } \\
\text { Change, Powder, Thermal, Fabrication, Freeform, }\end{array}$ \\
\hline Stratasys & & $\begin{array}{c}\text { Extrusion, Filament, Head, Spool, Assembly, Digital, } \\
\text { Layer, Prototyping }\end{array}$ \\
\hline
\end{tabular}

The technologies based on common keywords are essential to general 3D printing. Further, we can confirm the specific technologies of the competitive companies using their separate keywords. Next, we computed the frequency values of the keywords to know the technological trends based on keywords.

Next, we counted the frequency values of the keywords over year to know the technological trends based on keywords. Figure 10 shows the trends of "Pour", "Composition", "Imaging", and "Fabrication" in the technological keywords of 3D Systems.

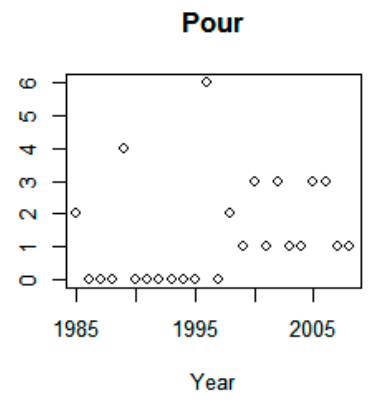

Imaging

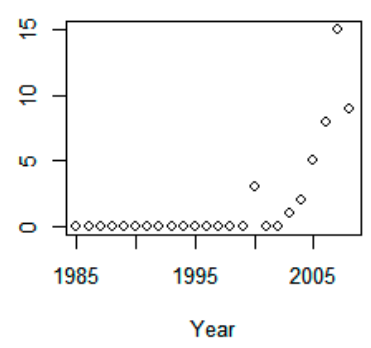

Composition

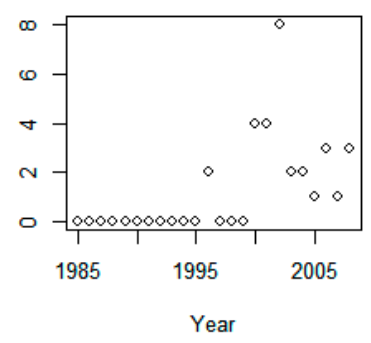

Fabrication

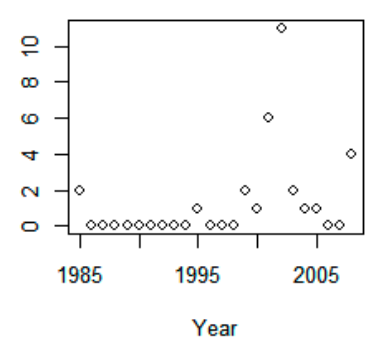

Figure 10. TS plots for 3D Systems: Pour, Composition, Imaging, Fabrication. 
We see that each keyword has its inherent trend. The TS trend of "Imaging" keyword is increasing rapidly. Thus, we know the importance of the technology based on "Imaging" will be increased continuously in the future. In this paper, we can also get more TS plots for entire 32 keywords of 3D Systems, and understand their TS trend like the case of "Imaging". Next, the TS plots of the keywords of "Extrusion", "Object", "Layer", and "Material" in the competitor of 3D Systems, Stratasys, are shown in Figure 11.

Extrusion

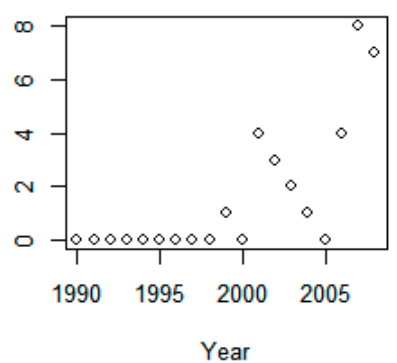

Layer

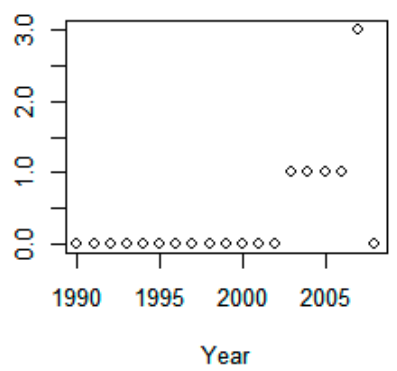

Object

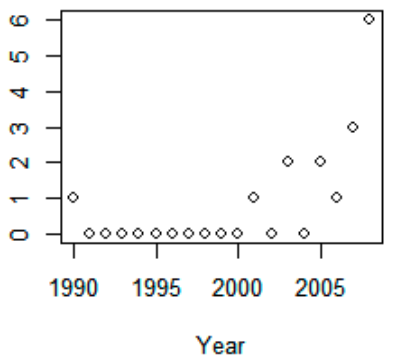

Material

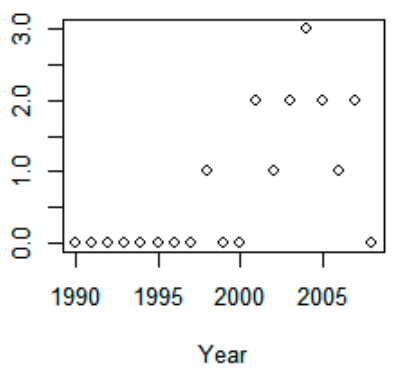

Figure 11. TS plots for 3D Systems: Extrusion, Object, Layer, Material.

We can find that the four TS plots have their distinct trends representing the technological keywords. In addition, we can show the all TS plots for the 20 Stratasys keywords. We found that each keyword had a distinct TS plot. The importance of a technology based on a keyword with a greater increasing trend than in the past is increasing. Using these results of TS plots, DTW assigns all keywords to similar groups. To compare the technological trends of the keywords detailed, we performed TSC using the TS plots of all keywords. In our TSC, we clustered all the keywords into similar group with a similar TS trend. In this paper, we show TSC results according to the number of clusters from two to five. First, we show the TSC results for 3D Systems in Figure 12.

When the number of clusters is two, the technologies of 3D Systems are divided into "Method" technologies and others. We also show the TSC result when the number of clusters is three. In this result, we know the technologies of 3D Systems are divided into two categories: "Method" technologies and "Modeling" technologies. The "Three_Dim" keyword is basic term of 3D printing technology, so we do not consider this keyword to be a technology cluster. Next, we present the keyword TSC result when the number of clusters is four. In Figure 12, we show the technologies of 3D Systems divided into "Method", "Modeling", and "Forming" technologies. To get a more detailed TSC result for 3D Systems' technologies, we perform TSC with five clusters. The keywords that are close to each other have a similar pattern in their technological trends. That is, we know that the technologies for the keywords showing a similar pattern were developed together. Next, we show the TSC results for Stratasys based on the number of clusters in Figure 13. 


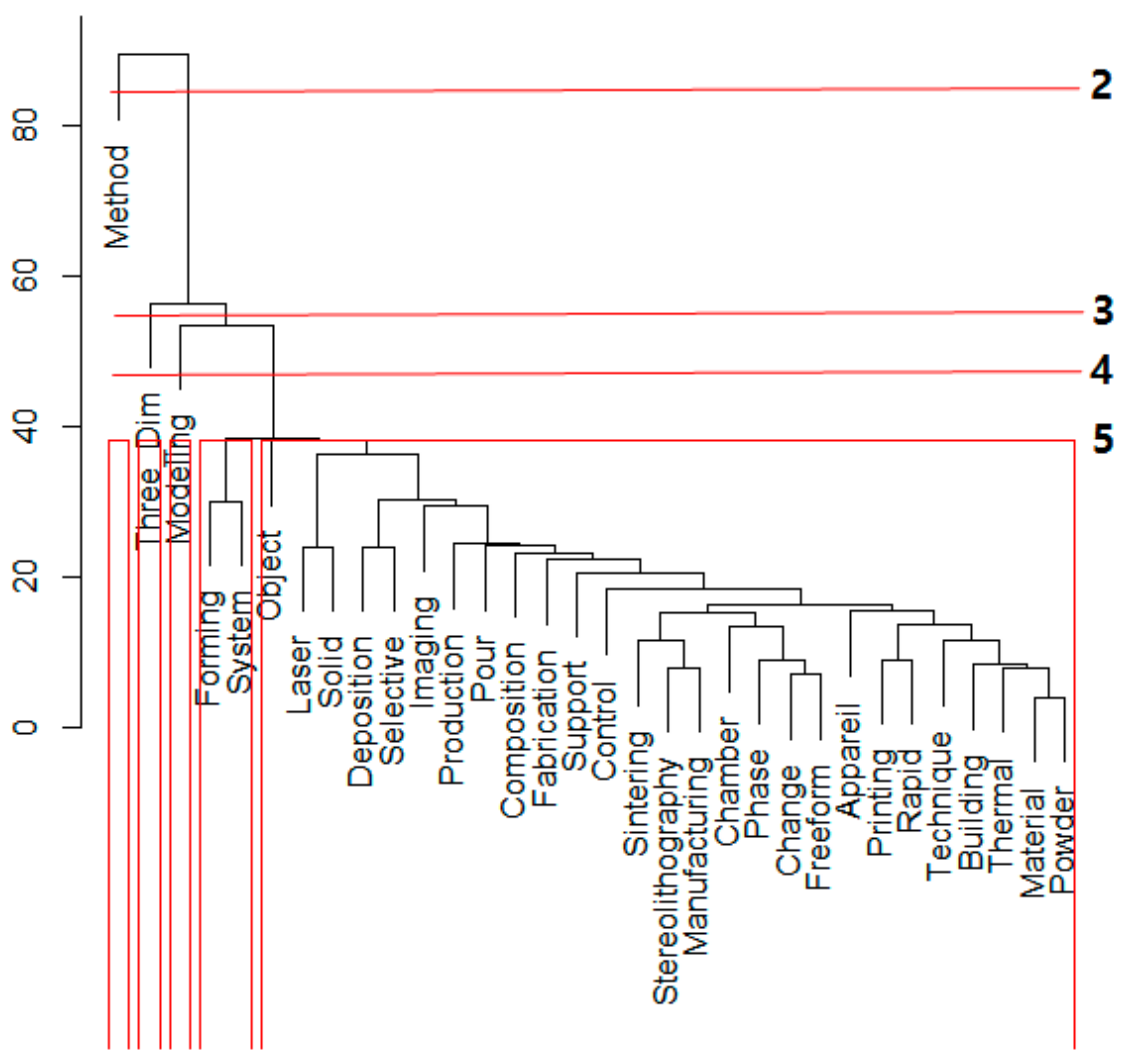

Figure 12. TSC for 3D Systems according to number of clusters.

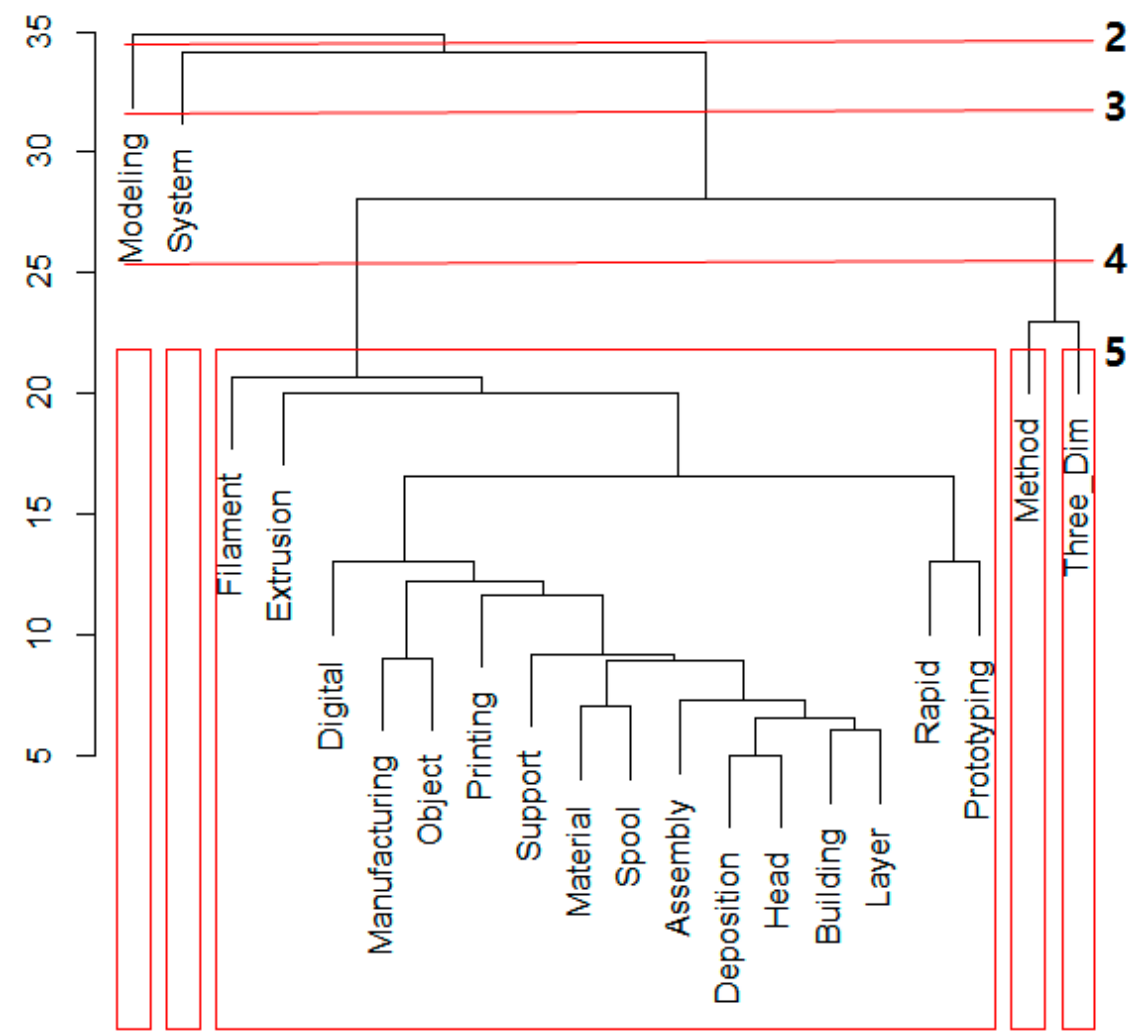

Figure 13. TSC for Stratasys according to number of clusters. 
When the number of clusters is two, the technologies held by Stratasys are divided into the technology groups of "Modeling" and "System". In addition, we show the TSC result with three clusters. The technologies held by Stratasys are divided into "Modeling", "System", and "Method" technologies. Thus, we know that these technological groups comprise the 3D printing technology held by Stratasys. We also show the TSC result for Stratasys when the number of clusters is four.

In Figure 13, we found the technologies of Stratasys were divided into four technology groups: "Modeling", "System", "Method", and "Filament". As we did for 3D Systems, we performed TSC with five clusters. The five categories of Stratasys' technologies for 3D printing are "Modeling", "System", "Method", "Filament", and "Three-Dim". Using the results of TSC, we can cluster the keywords representing technologies into similar groups, and we can find the associations between technologies related to 3D printing. Table 2 shows the TSC results for the two competitors according to the number of clusters.

Table 2. TSC results for 3D Systems and Stratasys.

\begin{tabular}{|c|c|c|}
\hline Number of Clusters & 3D Systems & Stratasys \\
\hline 2 & (Method), (Three_Dim) & (Modeling), (System) \\
\hline 3 & (Method), (Three_Dim), (Modeling) & (Modeling), (System), (Method) \\
\hline 4 & (Method), (Three_Dim), (Modeling), (Forming) & (Modeling), (System), (Method), (Filament) \\
\hline 5 & $\begin{array}{c}\text { (Method), (Three_Dim), (Modeling), (Forming), } \\
\text { (Object) }\end{array}$ & $\begin{array}{c}\text { (Modeling), (System), (Method), (Filament), } \\
\text { (Three_Dim) }\end{array}$ \\
\hline
\end{tabular}

From the results in Table 3, we found that "Modeling", "Method", and "Three_Dim" technologies are common technologies between the two competitors. However, the "Forming" and "Object" technologies are more developed by 3D Systems than by Stratasys, and the "System" and "Filament" technologies are more important for Stratasys than for 3D Systems. Next, we performed SNA for another analysis of technological relationships. Next, we performed SNA for another analysis of technological competition between two companies. We constructed the SNA graphs for the technologies of 3D Systems and Stratasys respectively, and compared the graphs. Figure 14 shows the SNA graph for 3D Systems' technology.

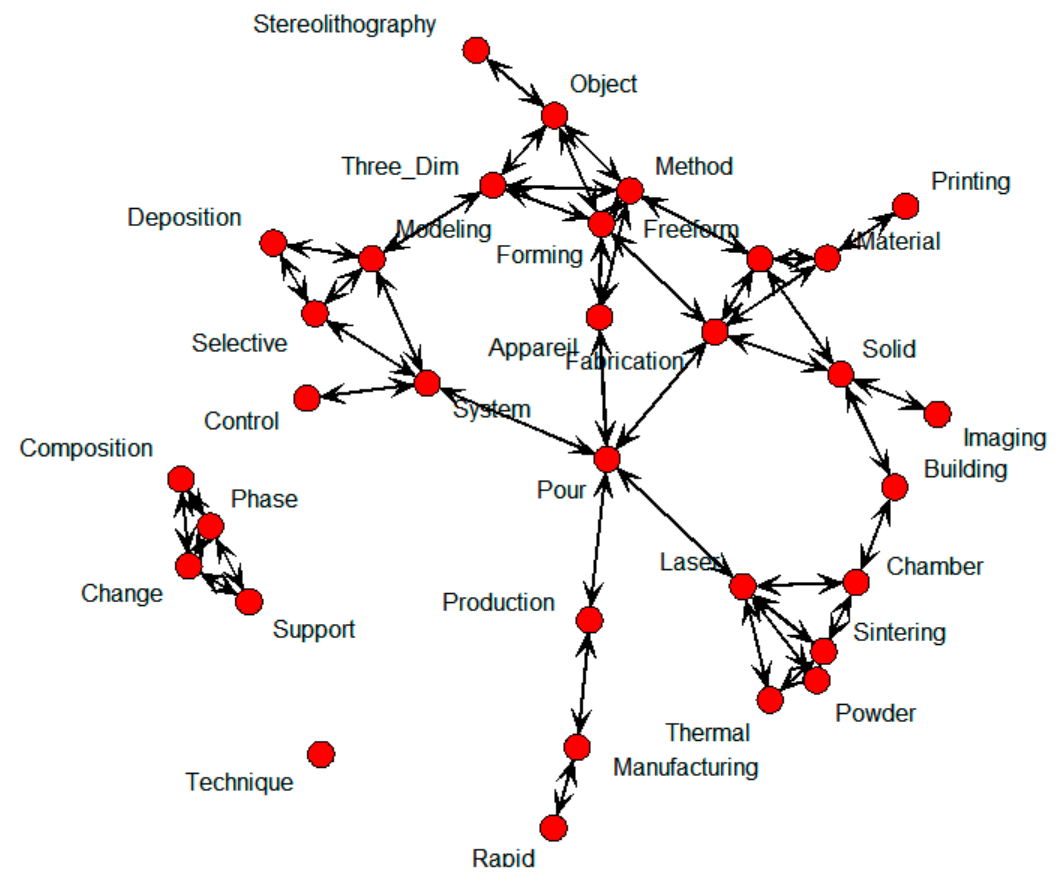

Figure 14. SNA graph for 3D Systems. 
Using the result of this SNA graph, we found the technological associations of 3D printing technologies for 3D Systems. For example, the keyword directly connected to "Printing" is "Material". That is, printing technology is influenced by technology dealing with material directly. The keywords (Material, Freeform, Method, Object, etc.) located within the "Three_Dim" and "Printing" categories represent intermediary technologies connecting two technologies based on "Three_Dim" and "Printing". In addition, we can understand the diverse associations of the technologies held by 3D Systems using the SNA result. Figure 15 shows the SNA result for Stratasys.

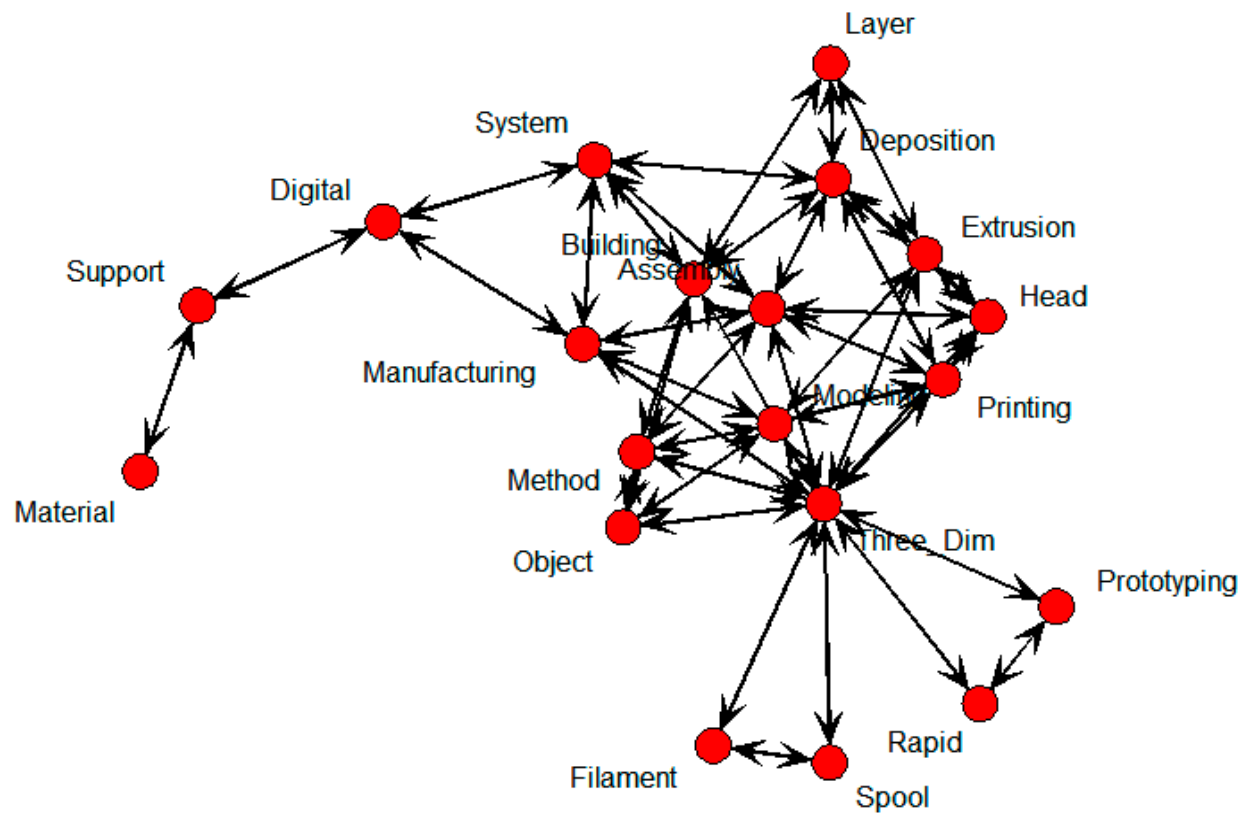

Figure 15. SNA graph for Stratasys.

Unlike in the SNA graph for 3D Systems, the keywords of "Three_Dim" and "Printing" are connected to each other directly for Stratasys. Thus, we know that the development of 3D printing technology at Stratasys is more efficient than that at 3D Systems. In contrast, Stratasys has developed technologies with greater emphasis on system modeling for 3D printing than has 3D Systems. We also performed statistical testing of SNA results. Table 4 shows the SNA graph tests of two companies.

Table 3. SNA graph tests of 3D Systems and Stratasys.

\begin{tabular}{ccc}
\hline Graph Test & 3D Systems & Stratasys \\
\hline Test value & 0.9027 & 0.9235 \\
\hline$p(\mathrm{~T} \geq$ test $)$ & 0.6140 & 0.9140 \\
\hline$p(\mathrm{~T}<$ test $)$ & 0.3860 & 0.0860 \\
\hline
\end{tabular}

Table 4. Technological competition for sustainability.

\begin{tabular}{|c|c|c|c|}
\hline Method & & 3D Systems & Stratasys \\
\hline \multirow{2}{*}{ TSC } & \multicolumn{3}{|c|}{ 3D, method, modeling } \\
\hline & & forming, object & filament, system \\
\hline \multirow[t]{2}{*}{ SNA } & $3 \mathrm{D}$ & modeling, object, forming, method & $\begin{array}{l}\text { rapid, filament, prototype, object, method, printing, } \\
\text { spool, extrusion }\end{array}$ \\
\hline & Printing & material & deposition, assembly, head, modeling, 3D \\
\hline
\end{tabular}


The test values of the two competitors are nearly the same statistics. Thus, we know the SNA results of this case study have validity to compare two companies. From the results of TSC and SNA, we illustrate the technological competition for sustainability of 3D print in Table 4.

In the TSC result, "3D", "method", and "modeling" are all common keywords of two companies. Thus, the technologies based on "3D", "method", and "modeling" are common sustainable technologies of two companies. The keywords of "forming" and "object" are representative to 3D Systems, we drive that the technology based on "forming" and "object" are a sustainable technology for 3D systems. Stratasys has the keywords of "filament" and "system" as its representative keywords. The technology depended on "filament" and "system" can be selected as sustainable technology of Stratasys. In the SNA result, we show the connected keywords to main keywords (3D and Printing) of 3D printing technology. "modeling", "object", "forming", and "method" are connected to "3D", and "material" is connected to "printing" in 3D Systems. The Stratasys has more keywords connected to "3D" and "Printing" than 3D Systems. From the result in Table 4, we conclude that the sustainable technology of 3D Systems is to form object using diverse materials. The Stratasys has the sustainability of technology based on extrusion and deposition system. In addition, the technological scope of Stratasys is larger than 3D Systems, because the numbers of keywords connected to "3D" and "Printing" of Stratasys are larger than 3D Systems. From the previous results in this paper, we constructed sustainable technology diagram (Figure 16).

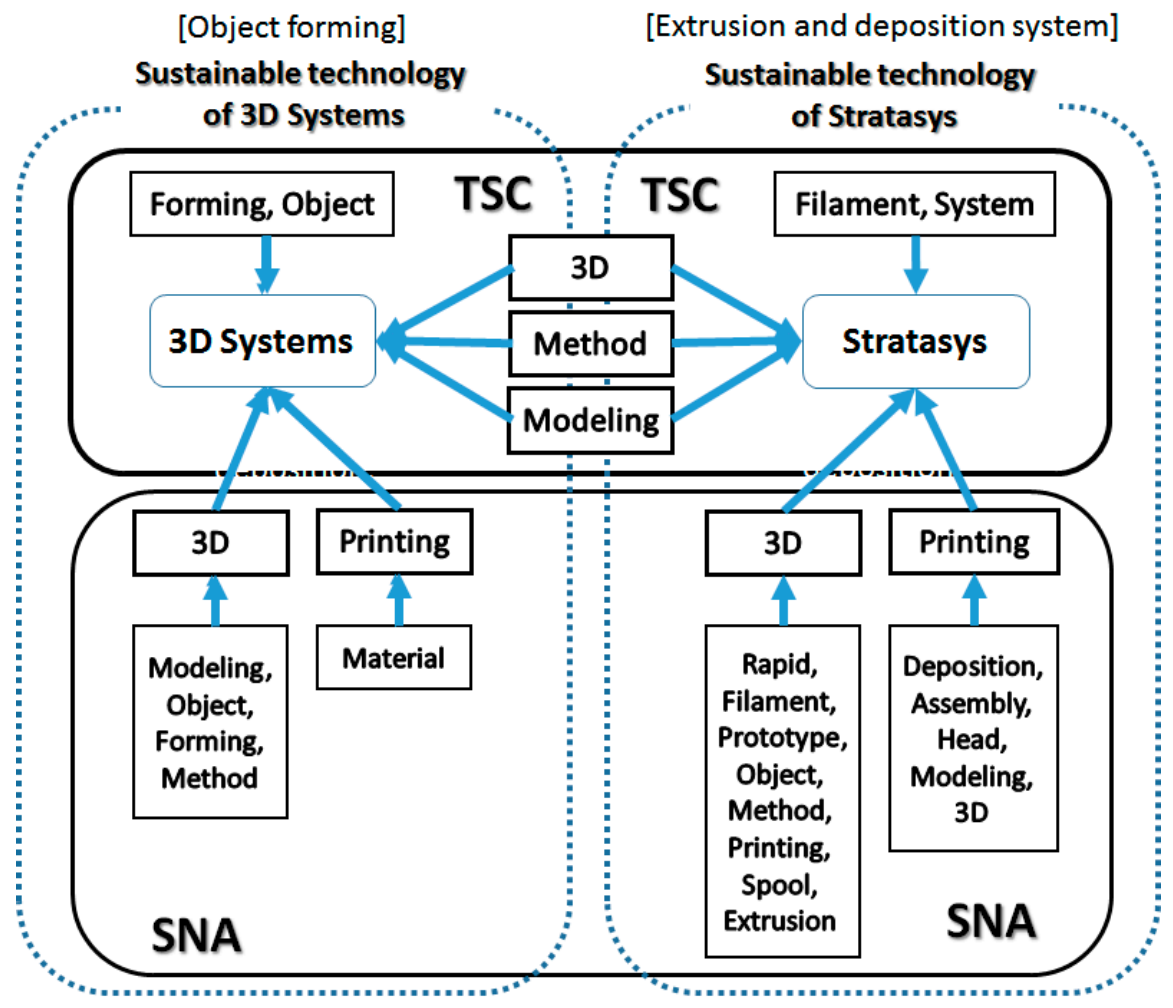

Figure 16. Sustainable technology diagram.

For each company, we built the technological relations between patent keywords according to TSC and SNA. In addition, we combined the results of TSC with SNA in Figure 16. Lastly, we extracted the sustainable technology areas from the diagram. We had help from the expert group related to 3D printing technology in the KISTA [55] to define the specific technologies for the sustainability of two competitors. In conclusion, the sustainable technologies of 3D Systems and Stratasys are defined as "object forming", and "extrusion and deposition system", respectively. Numerous research institutions and consulting firms around the world, including PwC, Lockheed Martin, Boeing, Google, 
and Mckinsey, believe that 3D printing technology is one of the key technologies for humanity's future sustainability [38]. Thus, we selected 3D printing technology as a technology field for performance evaluation of the proposed method. We also compared the sustainable technologies of two competitors (3D Systems and Stratasys) related to 3D printing technology. From the result of our sustainable technology diagram (Figure 16), we conclude that the technology of "object forming" is a necessary technology for 3D Systems' sustainability, and Stratasys needs the technology of "extrusion and deposition system" for its sustainability.

\section{Conclusions}

In this paper, we proposed a methodology of technology analysis between competitors from the point of sustainable technology. The traditional approaches for competitors' technology analysis were based on the knowledge and experience of domain experts; for example, the Delphi methodology is used to survey a domain expert group using a questionnaire to ask about their respective views about the technology. The result of the Delphi can fluctuate and be inconsistent because the method is dependent upon the experts' professionalism. In comparison, we proposed an objective and consistent methodology for sustainable technology analysis between competitors. Through the results of SNA and TSC using patent data for two competitors (3D Systems and Stratasys), we compared their technological development for sustainability. We knew that Stratasys has more focused technology than 3D Systems in the technological area of 3D printing methods. In addition, the technological competitiveness of 3D Systems is a result of technologies based on "Forming" and "Object". Thus, 3D Systems should research and develop these technological areas to improve the sustainable technological competition. In addition, "System", "Extrusion" and "Deposition" technologies are a sustainable technology area in which Stratasys can improve its technological competiveness. Our research result contributes to the $R \& D$ planning of the companies related to 3D printing technology as well as 3D Systems and Stratasys.

This paper contributes to stable and consistent analyses of sustainable technologies between competitors using objective data (patent documents) and quantitative methods (TSC and SNA). Our research can be applied to many comparisons of technology analysis between competitors, such as Apple, Google, and Samsung. In this paper, we did not consider the opinions of experts and specialists related to 3D printing technology. If we can combine the results of this paper with expert opinions, we can expect a more accurate and valid result for sustainable technology analysis between competitors. Thus, in our future work, we will apply opinion mining [56], sentiment analysis [57], and topic model [58,59] to our methodology for sustainable technology analysis. This paper dealt with a more efficient way of finding sustainability in a specific technology field by introducing a new time concept that was not covered in the existing quantitative analysis methods for selecting sustainable technologies. Therefore, this study will contribute to finding the sustainable technology in most technical fields and thereby establishing R \& D plan efficiently.

Acknowledgments: This research was supported by the Basic Science Research Program through the National Research Foundation of Korea (NRF) funded by the Ministry of Education (NRF-2015R1D1A1A01059742).

Author Contributions: Sangsung Park designed this research and collected the data set for the experiment. Sunghae Jun analyzed the data to show the validity of this paper and wrote the paper and performed all the research steps. In addition, all authors have cooperated with each other for revising the paper.

Conflicts of Interest: The authors declare no conflict of interest.

\section{References}

1. Mulder, K.; Ferrer, D.; Lente, H.V. What Is Sustainable Technology? Perceptions, Paradoxes and Possibilities; Greenleaf Publishing: Bradford, UK, 2011.

2. Roper, A.T.; Cunningham, S.W.; Porter, A.L.; Mason, T.W.; Rossini, F.A.; Banks, J. Forecasting and Management of Technology; John Wiley \& Sons: New York, NY, USA, 2011.

3. Kim, S.; Jang, D.; Jun, S.; Park, S. A Novel Forecasting Methodology for Sustainable Management of Defense Technology. Sustainability 2015, 7, 16720-16736. [CrossRef] 
4. Choi, J.; Jang, D.; Jun, S.; Park, S. A Predictive Model of Technology Transfer using Patent Analysis. Sustainability 2015, 7, 16175-16195. [CrossRef]

5. Park, S.; Lee, S.; Jun, S. A Network Analysis Model for Selecting Sustainable Technology. Sustainability 2015, 7, 13126-13141. [CrossRef]

6. Choi, J.; Jun, S.; Park, S. A Patent Analysis for Sustainable Technology Management. Sustainability 2016, 8, 1-13. [CrossRef]

7. Kim, J.; Lee, J.; Kim, G.; Park, S.; Jang, D. A Hybrid Method of Analyzing Patents for Sustainable Technology Management in Humanoid Robot Industry. Sustainability 2016, 8, 474. [CrossRef]

8. Park, S. Analysis of Patents Issue Report-3D Printing; HIS Electronics \& Media: Englewood, CO, USA, 2013.

9. Kwak, K.; Park, S. Technology trend analysis of global 3D printing industry. J. KSME 2013, 53, 58-64.

10. Geum, Y.; Lee, S.; Yoon, B.; Park, Y. Identifying and evaluating strategic partners for collaborative R\&D: Index-based approach using patents and publications. Technovation 2013, 33, 211-224.

11. Goetze, C. An empirical enquiry into co-patent networks and their stars: The case of cardiac pacemaker technology. Technovation 2010, 30, 436-446. [CrossRef]

12. Jeong, Y.; Yoon, B. Development of patent roadmap based on technology roadmap by analyzing patterns of patent development. Technovation 2015, 39, 37-52. [CrossRef]

13. Lee, S.; Yoon, B.; Park, Y. An approach to discovering new technology opportunities: Keyword-based patent map approach. Technovation 2009, 29, 481-497. [CrossRef]

14. Jun, S. Central technology forecasting using social network analysis. Commun. Comput. Inf. Sci. 2012, 340, 1-8.

15. Jun, S.; Park, S. Examining technological innovation of Apple using patent analysis. Ind. Manag. Data Syst. 2013, 113, 890-907. [CrossRef]

16. Otte, E.; Rousseau, R. Social network analysis: a powerful strategy, also for the information sciences. J. Inf. Sci. 2002, 28, 441-453. [CrossRef]

17. Sternitzke, C.; Bartkowski, A.; Schramm, R. Visualizing patent statistics by means of social network analysis tools. World Pat. Inf. 2008, 30, 115-131. [CrossRef]

18. Jun, S. Technology network model using bipartite social network analysis. Commun. Comput. Inf. Sci. 2012, $340,28-35$.

19. Jun, S.; Choi, J. A technology analysis model using dynamic time warping. J. Korea Soc. Comput. Inf. 2015, 20, 113-120.

20. Chifu, A.G.; Hristea, F.; Mothe, J.; Popescu, M. Word sense discrimination in information retrieval: A spectral clustering-based approach. Inf. Process. Manag. 2015, 51, 16-31. [CrossRef]

21. Jun, S.; Park, S.S.; Jang, D.S. Document clustering method using dimension reduction and support vector clustering to overcome sparseness. Expert Syst. Appl. 2014, 41, 3204-3212. [CrossRef]

22. Liu, C.L.; Hsaio, W.H.; Lee, C.H.; Chen, C.H. Clustering tagged documents with labeled and unlabeled documents. Inf. Process. Manag. 2013, 49, 596-606. [CrossRef]

23. Dao, V.T.; Zmud, B. The Influence of Technology-Related, Market-Related, and Standards-Related Strategic Signaling During a Standards War. IEEE Trans. Eng. Manag. 2015, 62, 300-310. [CrossRef]

24. Jun, S.; Park, S.; Jang, D. Technology Forecasting using Matrix Map and Patent Clustering. Ind. Manag. Data Syst. 2012, 112, 786-807. [CrossRef]

25. Lai, H.C.; Weng, C.S. Accessing External Technological Knowledge for Technological Development: When Technological Knowledge Distance Meets Slack Resources. IEEE Trans. Eng. Manag. 2014, 61, 80-89. [CrossRef]

26. Tseng, Y.; Lin, C.; Lin, Y. Text mining techniques for patent analysis. Inf. Process. Manag. 2007, 43, $1216-1247$. [CrossRef]

27. Chen, D.; Lin, W.C.; Huang, M. Using essential patent index and essential technological strength to evaluate industrial technological innovation competitiveness. Scientometrics 2007, 71, 101-116. [CrossRef]

28. Chen, Y.L.; Chiu, Y.T. An IPC-based vector space model for patent retrieval. Inf. Process. Manag. 2011, 47, 309-322. [CrossRef]

29. Chen, Y.L.; Chang, Y.C. A three-phase method for patent classification. Inf. Process. Manag. 2012, 48, 1017-1030. [CrossRef]

30. Guan, J.; Chen, Z. Patent collaboration and international knowledge flow. Inf. Process. Manag. 2012, 48, 170-181. [CrossRef]

31. Hunt, D.; Nguyen, L.; Rodgers, M. Patent Searching Tools E Techniques; Wiley: New York, NY, USA, 2007.

32. Berry, M.W.; Kogan, J. Text Mining Applications and Theory; Wiley: New York, NY, USA, 2010. 
33. Feinerer, I. A Text Mining Framework in R and Its Applications. Ph.D. Thesis, Vienna University of Economics and Business Administration, Vienna, Austria, 2008.

34. Jun, S. A technology forecasting method using text mining and visual apriori algorithm. Appl. Math. Inf. Sci. Int. J. 2014, 8, 35-40. [CrossRef]

35. Sun, Y.; Lu, Y.; Wang, T.; Ma, H.; He, G. Pattern of patent-based environmental technology innovation in China. Technol. Forecast. Soc. Chang. 2008, 75, 1032-1042. [CrossRef]

36. Feinerer, I.; Hornik, K.; Meyer, D. Text mining infrastructure in R. J. Stat. Softw. 2008, 25, 1-54. [CrossRef]

37. Butts, C.T. Social network analysis with SNA. J. Stat. Softw. 2008, 24, 1-51. [CrossRef]

38. D'Aveni, R. The 3-D printing revolution. Harv. Bus. Rev. 2015, 93, 40-48.

39. Bommel, V.T.; Mahieu, R.J.; Nijssen, E.J. Technology trajectories and the selection of optimal R\&D project sequences. IEEE Trans. Eng. Manag. 2014, 61, 669-680.

40. Liao, T.W. A clustering procedure for exploratory mining of vector time series. Pattern Recognit. 2007, 40, 2550-2562. [CrossRef]

41. Zhang, X.; Liu, J.; Du, Y.; Lv, T. A novel clustering method on time series data. Expert Syst. Appl. 2011, 38, 11891-11900. [CrossRef]

42. Zhao, Y.R. Data Mining-Examples and Case Studies; Academic Press: Elsevier, Amsterdam, The Netherlands, 2013.

43. Giorgino, T. Package 'dtw'-Dynamic Time Warping Algorithms. 2014. Available online: dtw.r-forge.rproject.org (accessed on 15 October 2016).

44. Keogh, E.J.; Pazzani, M.J. Derivative dynamic time warping. In Proceedings of the 2001 SIAM International Conference on Data Mining, Chicago, IL. USA, 2011; pp. 1-11.

45. Salvador, S.; Chan, P. Toward accurate dynamic time warping in linear time and space. Intell. Data Anal. 2007, 11, 561-580.

46. Mann, D.L. Better technology forecasting using systemic innovation methods. Technol. Forecast. Soc. Chang. 2003, 70, 779-795. [CrossRef]

47. Trappey, A.J.C.; Trappey, C.V.; Wu, C.; Lin, C. A patent quality analysis for innovative technology and product development. Adv. Eng. Inf. 2012, 26, 26-34. [CrossRef]

48. Feinerer, I.; Hornik, K. Text Mining Package-Package Tm. In CRAN R-Project; CRAN R-Project: Vienna, Austria, 2017.

49. Butts, C.T. Tools for Social Network Analysis-Package Sna. In CRAN R-Project; CRAN R-Project: Vienna, Austria, 2017.

50. R Development Core Team. R: A Language and Environment for Statistical Computing; R Foundation for Statistical Computing: Vienna, Austria, 2017.

51. USPTO. United States Patent and Trademark Office. Available online: http://www.uspto.gov/ (accessed on 1 June 2015).

52. WIPSON. WIPS Corporation. Available online: http://www.wipson.com/ (accessed on 24 July 2015).

53. Gridlogics Technologies Pvt. Ltd. Patent iNSIGHT Pro. 3D Printing Technology Insight Report; Gridlogics Technologies Pvt. Ltd.: Mumbai, India, 2014.

54. UK Intellectual Property Office Patent Informatics Team. 3D Printing: A Patent Overview; Intellectual Property Office: Newport, UK, 2013.

55. KISTA. Korea Intellectual Property Strategy Agency. 2016. Available online: www.kista.or.kr (accessed on 1 December 2016).

56. Severyn, A.; Moschitti, A.; Uryupina, O.; Plank, B.; Filippova, K. Multi-lingual opinion mining on youtube. Inf. Process. Manag. 2016, 52, 46-60. [CrossRef]

57. Medhat, W.; Hassan, A.; Korashy, H. Sentiment analysis algorithms and applications: A survey. Ain Shams Eng. J. 2014, 5, 1093-1113. [CrossRef]

58. Blei, D.M. Probabilistic topic models. Commun. ACM 2012, 55, 77-84. [CrossRef]

59. Park, S.; Lee, W.; Moon, I.C. Associative topic models with numerical time series. Inf. Process. Manag. 2015, 51, 737-755. [CrossRef]

(C) 2017 by the authors. Licensee MDPI, Basel, Switzerland. This article is an open access article distributed under the terms and conditions of the Creative Commons Attribution (CC BY) license (http://creativecommons.org/licenses/by/4.0/). 